\title{
Icariin ameliorates dexamethasone-induced bone deterioration in an experimental mouse model via activation of microRNA-186 inhibition of cathepsin K
}

\author{
YONGSHENG MA, HAO YANG and JUNQING HUANG \\ Department of Orthopaedics, The Second Affiliated Hospital of Henan University of \\ Traditional Chinese Medicine, Zhengzhou, Henan 450002, P.R. China
}

Received February 29, 2016; Accepted February 15, 2017

DOI: $10.3892 / \mathrm{mmr} .2017 .8065$

\begin{abstract}
The present study aimed to investigate bone deterioration in glucocorticoid-induced osteoporosis (GIOP) mice, and the anti-osteoporosis effect and underlying molecular mechanism of icariin. Dexamethasone (DSM) treatment was demonstrated to facilitate the induction of hypercalciuria in GIOP mice. Icariin treatment reversed the dexamethasone (DXM)-induced disequilibrium of calcium homeostasis and bone resorption, and increased serum alkaline phosphatase, tartrate resistant acid phosphatase, osteocalcin and deoxypyridinoline. Haematoxylin and eosin staining revealed an increase in disconnections and separation in the trabecular bone network of the tibial proximal metaphysis, in the GIOP group. Icariin treatment reversed the DXM-induced trabecular deleterious effects, and stimulated bone remodeling in GIOP mice. Furthermore, the results demonstrated that the mRNA and protein expression of cathepsin $\mathrm{K}$ were significantly increased in GIOP mice, compared with the control group. Icariin treatment may suppress the expression of cathepsin $\mathrm{K}$ in the tibia of GIOP mice. The levels of microRNA (miR)-186 were markedly reduced in the tibia of GIOP mice compared with control group; however, this was inhibited by icariin treatment. Bioinformatics analysis demonstrated that miR-186 regulates cathepsin $\mathrm{K}$ via binding to the upstream 3'-untranslated region. Furthermore, transfection with miR-186 mimics resulted in inhibition of cathepsin K expression, whereas miR-186 inhibitors facilitated cathepsin $\mathrm{K}$ expression in osteoclasts. In conclusion, the present study demonstrated the protective effects of icariin against bone deteriorations in the experimental GIOP
\end{abstract}

Correspondence to: Dr Yongsheng Ma, Department of Orthopaedics, The Second Affiliated Hospital of Henan University of Traditional Chinese Medicine, 6 Dongfeng Road, Zhengzhou, Henan 450002, P.R. China

E-mail: ma_yssh@163.com

Key words: osteoporosis, icariin, dexamethasone, microRNA-186, cathepsin $\mathrm{K}$ mice, and the underlying mechanism was mediated, at least partially, via activation of miR-186-mediated suppression of cathepsin $\mathrm{K}$. These results provide evidence to support the use of icariin as a therapeutic approach in the management of glucocorticoid-induced bone loss, and the disequilibrium of calcium homeostasis.

\section{Introduction}

Glucocorticoid-induced osteoporosis (GIOP) is a severe complication of prolonged systemic glucocorticoid use. GIOP-mediated bone loss, and the associated risk of fracture, is a relatively common disorder and the most prevalent type of secondary osteoporosis (1). It is believed that glucocorticoids may cause rapid bone loss, reduced bone formation and increased bone resorption, in vitro and in vivo $(2,3)$. The therapeutic use of low doses of oral glucocorticoids and mild endogenous hypercortisolism may also be associated with bone loss (4). However, patients treated with glucocorticoids are not often evaluated and treated for this problem. Therefore, the exploration of a novel and effective adjuvant therapy is required.

Icariin has been identified as a flavonoid isolated from Herba Epimedii (Epimedium brevicornum), which is the most commonly used Chinese herbal medicine in the treatment of sexual dysfunction and osteoporosis $(5,6)$. The anti-osteoporotic role of icariin has been verified in ovariectomized rats $(7,8)$ and glucocorticoid-induced osteoporotic rats (9). A 24-month randomized double-blind placebo-controlled clinical trial indicated that an Epimedium-derived phytoestrogen flavonoid preparation containing $60 \mathrm{mg}$ icariin, $15 \mathrm{mg}$ daidzien and $3 \mathrm{mg}$ genistein was able to limit bone loss in late postmenopausal women (10). Notably, molecular mechanism studies have suggested that icariin may induce osteoblast proliferation, differentiation and mineralization via estrogen receptor-mediated extracellular-signal related kinase and c-Jun N-terminal kinase signal activation (11), and may regulate the osteogenic differentiation of bone mesenchymal stem cells in a mitogen activated protein kinase-dependent manner (12). Furthermore, icariin, as an inhibitor of cathepsin $\mathrm{K}$, suppresses cartilage and bone degradation in 
mice with collagen-induced arthritis (13). Previous study has indicated that cathepsin $\mathrm{K}$ serves an important role in the pathogenesis of rheumatoid arthritis (14). Cathepsin $\mathrm{K}$ is expressed by osteoclasts during bone resorption, and serves as the major collagenase responsible for organic bone matrix degradation during the bone remodeling process (15). Excessive cathepsin $\mathrm{K}$ is a key element in the pathogenesis of postmenopausal osteoporosis and other skeletal disorders; therefore, cathepsin $\mathrm{K}$ may be a valuable target in the development of novel therapeutic intervention strategies (16). The expression of cathepsin $\mathrm{K}$ and the effects of icariin in GIOP animal models remains largely unknown.

MicroRNAs (miRNAs) are a class of small noncoding (18-25 nucleotides), endogenous RNA molecules that regulate the translation of messenger RNAs (mRNAs) by attaching to the target 3'-untranslated region (3'-UTR) of mRNAs $(17,18)$. The key roles of miRNAs involve regulation of cell proliferation, differentiation and apoptosis of several cell types, including osteoblasts and osteoclasts $(19,20)$. A growing number of studies have demonstrated pathogenic alterations in various tissues that may be linked to abnormal miRNA expression, including the regulation of bone development $(21,22)$. The present study hypothesized that a miRNA mechanism may be involved in icariin-mediated inhibition of secondary osteoporosis in mice, by targeting cathepsin K. This study aimed to improve the understanding of the anti-osteoporotic action and underlying molecular mechanism of icarrin, which may facilitate the treatment of secondary osteoporotic patients.

\section{Materials and methods}

Animal treatment. All procedures were evaluated and approved by the ethics committee of the Henan University of Traditional Chinese Medicine (Henan, China). A total of 48 male C57BL/6J mice (age, 8 weeks; weight, $20 \pm 2 \mathrm{~g}$ ) were obtained from Slac Laboratory Animal (Shanghai, China; www.slaccas.com) and were allowed to acclimate to the environment for 1 week. The mice were given free access to food and tap water and were caged individually under controlled temperature $\left(23 \pm 2^{\circ} \mathrm{C}\right)$ and humidity $(55 \pm 5 \%)$ with an artificial 12-h light/dark cycle. The mice were randomly divided into four groups: Vehicle group $(n=12)$ were injected with normal saline; dexamethasone (DXM), where mice were injected intramuscularly with $5 \mathrm{mg} / \mathrm{kg}$ body weight DXM three times weekly, for 12 weeks $(n=12)$; low dose icariin GIOP, mice received oral icariin at a dose of $10 \mathrm{mg} / \mathrm{kg} /$ day for 12 weeks (Icariin $+\mathrm{L}, \mathrm{n}=12$ ) combined with DXM; high dose icariin GIOP, mice received oral icariin at a dose of $100 \mathrm{mg} / \mathrm{kg} / \mathrm{day}$, for 12 weeks combined with DXM (Icariin + H, $n=12$ ).

Cell culture. Human embryonic kidney HEK293T cells [cat. no. CRL-11268; American Type Culture Collection (ATCC), Manassas, VA, USA] were maintained in Dulbecco's modified Eagle's medium (DMEM; Thermo Fisher Scientific, Inc., Waltham, MA, USA) supplemented with 10\% (v/v) fetal bovine serum (FBS; Sigma-Aldrich; Merck Millipore, Darmstadt, Germany), $100 \mathrm{U} / \mathrm{ml}$ penicillin, and $100 \mathrm{mg} / \mathrm{ml}$ streptomycin, in a $5 \% \mathrm{CO}_{2}$ atmosphere at $37^{\circ} \mathrm{C}$.
Serum and urine chemistries. Mice were placed in metabolic cages for $24 \mathrm{~h}$ to collect and recorded the volume of urine at week 12. Blood were collected after the animals were sacrificed at week 12 by intraperitoneal injection of sodium pentobarbital (2\%; 200 mg/kg; cat. no. P3761; Sigma-Aldrich; Merck Millipore). The concentrations of calcium ( $\mathrm{Ca}$, absorbance was measured at $490 \mathrm{~nm}$ ) and creatinine $(\mathrm{Cr}$, absorbance was measured at $630 \mathrm{~nm}$ ) in serum and urine were measured by standard colorimetric methods, using a microplate reader (BioTek Instruments, Winooski, VT, USA). The urinary $\mathrm{Ca}$ level was corrected against the urinary $\mathrm{Cr}$ concentration. Serum levels of alkaline phosphatase (ALP, cat. no. P0321; Beyotime Institute of Biotechnology, Haimen, China), tartrate resistant acid phosphatase (TRAP, cat. no. P0332; Beyotime Institute of Biotechnology), osteocalcin (OCN, cat. no. 60-1305; Immutopics, Inc., San Clemente, CA, USA) and deoxypyridinoline (DPD, cat. no. Ek-M20919; EK-Bioscience, Biotechnology Co., Ltd., Shanghai, China) were detected using mouse bioactive ELISA assays and measured using a SpectraMax M5 ELISA plate reader (Molecular Devices, LLC, Sunnyvale, CA, USA).

Osteoclast formation in RAW264.7 cells. RAW264.7 cells (cat. no. TIB 71; ATCC) were cultured in $\alpha$-MEM $+10 \%$ FBS (Sigma-Aldrich; Merck Millipore) and were plated in 6-well dishes at $1 \times 10^{5}$ cells/well and were grown for 5 days with RANKL (20 ng/ml, cat. no. 462-TR; R\&D Systems, Minneapolis, MN, USA) induction of osteoclastogenesis, as previously described (23).

Caspase- 3 activity assay. Osteoclast $\left(5 \times 10^{4}\right)$ lysates were prepared in $2 \mathrm{ml}$ of lysis buffer, containing $25 \mathrm{mM}$ HEPES, $\mathrm{pH}$ 7.5,5 mM EDTA and $1 \mathrm{mM}$ EGTA (all from Sigma-Aldrich; Merck Millipore), $5 \mathrm{mM} \mathrm{MgCl}_{2}$ and $10 \mathrm{mM}$ sucrose (both from Thermo Fisher Scientific, Inc.), $5 \mathrm{mM}$ dithiothreitol (DTT), $1 \%$ 3-[-(3-chloramidopropyl) dimethylammonio]-1-propanesulfonic acid (CHAPS), protease inhibitor cocktail $(10 \mu \mathrm{l} / \mathrm{ml})$, and $1 \mathrm{mM}$ PMSF (all from Sigma-Aldrich; Merck Millipore). After $30 \mathrm{~min}$ incubation at $0^{\circ} \mathrm{C}$, cell lysates were centrifuged at $12,000 \mathrm{x} \mathrm{g}$ for $15 \mathrm{~min}$ at $4^{\circ} \mathrm{C}$, and the supernatants were collected. The activity of caspase- 3 was determined using the caspase-3 activity assay kit (cat. no. C1116; Beyotime Institute of Biotechnology), according to the manufacturer's instructions. Release of p-nitroaniline was measured at a wavelength of $405 \mathrm{~nm}$ using a SpectraMax M5 ELISA plate reader, according to the manufacturer's instructions.

Quantification of apoptosis by flow cytometry. Quantitative assessment of apoptotic osteoclasts $\left(5 \times 10^{4}\right)$ was performed by terminal deoxynucleotidyl transferase-mediated deoxyuridine triphosphate nick end labeling (TUNEL) method, which examines DNA-strand breaks during apoptosis, using an ApoAlert $^{\mathrm{TM}}$ DNA Fragmentation Assay kit (BD Biosciences, Franklin Lakes, NJ, USA). Cells were trypsinized, fixed with $4 \%$ paraformaldehyde and permeabilized with $0.1 \%$ Triton X-100 in $0.1 \%$ sodium citrate. The cells were washed with PBS three times and subsequently incubated with the reaction mixture for $60 \mathrm{~min}$ at $37^{\circ} \mathrm{C}$. Cells were immediately analyzed using a FACScan flow cytometer equipped with the CellQuest version 5.1 (BD Biosciences). 
Bone histomorphology. The tibias were collected after the animals were sacrificed and were decalcified in 0.5 M EDTA $(\mathrm{pH}=8.0)$ and embedded in paraffin by standard histological procedures. Sections $(5 \mu \mathrm{m})$ were cut and stained with hematoxylin \& eosin and safranin $\mathrm{O}$, and were visualized under a Leica DM 2500 microscope (Leica Microsystems GmbH, Wetzlar, Germany). The trabecular bone microarchitecture of the proximal metaphysis of the tibia was measured using a SkyScan 1076 microtomography scanner (Bruker Corporation, Ettlingen, Germany), with a slice thickness of $22 \mu \mathrm{m}$. Bone morphometric parameters, including volumetric bone mineral density (BMD), bone volume over total volume (BV/TV), trabecula number (Tb.N), trabecula thickness (Tb.Th) and trabecula separation (Tb.Sp) were measured, as previously described (24). Femurs were placed in a 3-point bending configuration on an Instron Microtester 5848 system (Instron, Norwood, MA USA), to measure maximum load and stiffness, as previously described (25).

Transfection of miRNA-186 mimics and inhibitor. The 6-carboxyfluorescein-conjugated 2'-OMe-oligonucleotides were chemically synthesized and purified by high-performance liquid chromatography (GenePharma Co., Ltd., Shanghai, China). The 2'-OMe-miRNA (miR)-186 mimic was composed of RNA duplexes with the following sequence: 5'-CAAAG AAUUCUCCUUUUGGGCU-3'. The 2'-OMe-miR-1271 inhibitor sequence was: 5'-AGCCCAAAAGGAGAAUUC UUUG-3', and the 2'-Ome-scramble sequence was: 5'-CUU GGUCAAGUCGCGAUCCGUA-3'. Osteoclasts $\left(1 \times 10^{5}\right)$ were transfected using Lipofectamine 2000 ${ }^{\mathrm{TM}}$ (Invitrogen; Thermo Fisher Scientific, Inc., Waltham, MA, USA), at a final concentration of $100 \mathrm{nM}$. The culture medium was changed $24 \mathrm{~h}$ post transfection. Cells were harvested for analysis after $48 \mathrm{~h}$.

Luciferase reporter gene activity assay. The 3'-UTR of the cathepsin $\mathrm{K}$ gene containing the predicated target site for miR-186 was synthesized by GenePharma. The fragment was inserted into the multiple cloning site in the pMIR-REPORT luciferase miRNA expression reporter vector (Ambion; Thermo Fisher Scientific, Inc.). HEK293T cells $\left(5 \times 10^{4}\right.$; cat. no. CRL-11268; ATCC) were co-transfected with luciferase reporters containing cathepsin K 3'-UTR and miR-186 mimics using Lipofectamine 2000. The cell lysates were harvested following $48 \mathrm{~h}$ transfection, and the luciferase activity was measured using a dual luciferase reporter assay kit (cat. no. RG027; Beyotime Institute of Biotechnology) according to manufacturer's instructions.

Reverse transcription-quantitative PCR (RT-qPCR.) The tibias were crushed in liquid nitrogen and RNA extraction was performed using TRIzol ${ }^{\circledR}$ according to the manufacturer's instructions (Invitrogen; Thermo Fisher Scientific, Inc.). Synthesis of cDNA was performed by reverse transcription of $2 \mu \mathrm{g}$ total RNA, using Moloney murine leukemia virus reverse transcriptase (Invitrogen; Thermo Fisher Scientific, Inc.), with oligo (dT) $)_{15}$ primers (Fermentas; Thermo Fisher Scientific, Inc., Pittsburgh, PA, USA), according to the manufacturer's instructions. The first strand cDNAs served as the template for the qPCR and were performed using an ABI 7300 thermocycler (Applied Biosytems; Thermo Fisher Scientific, Inc.). GAPDH served as an internal control to determine the relative expression of the target genes. The reaction conditions were set according to the kit protocol. The level of miR-186 was quantified using a mirVana qRT-PCR miRNA Detection kit (Ambion; Thermo Fisher Scientific, Inc.) and SYBR-Green (cat. no. D7260; Beyotime Institute of Biotechnology). Following the cycling reactions were used: $95^{\circ} \mathrm{C}$ for $10 \mathrm{~min}$, followed by 40 cycles of $95^{\circ} \mathrm{C}$ for $15 \mathrm{sec}, 58^{\circ} \mathrm{C}$ for $30 \mathrm{sec}$, and $72^{\circ} \mathrm{C}$ for $30 \mathrm{sec}$. U6 served as the internal control. The quantitation cycle $(\mathrm{Cq})$ method (26) was used to determine relative expression levels. The PCR primers (Sangon Biotech, Co., Ltd., Shanghai, China) used in the present study were as follows: forward, 5'-CCA GGGCGTACGGAGGCCATT-3' and reverse, 5'-GACCAA ATTACGGCGTAGCCTC-3' for ALP; forward, 5'-AGCATA AGGGTCCAAGTCCAA-3' and reverse, 5'-TACCAAAAG CGGCGTAGTTA-3' for TRAP; forward, 5'-AGGCGGAGG TCGATGCCCCG-3' and reverse, 5'-CACGATGATGTCACC CTCGATGT-3' for cathepsin $\mathrm{K}$, forward, 5'-ACCGTGAGT TGTCCGTAGCATC-3' and reverse, 5'-CGTAAGGGTCCG ATACATCTC-3' for osteoprotegerin (OPG); forward, 5'-CGT AGGTAGCCGGGTAGGATC-3' and reverse, 5'-GCGTGG GAGCCTGATGCTCA-3' for receptor activator of nuclear factor- $\kappa \mathrm{B}$ ligand (RANKL); forward, 5'-GGCAGGTGCTTC AGAACTGG-3' and reverse, 5'-GTGGTGGCAGGTAGGTAT GG-3' for Runt-related transcription factor 2 (Runx2); forward, 5'-TGAGCTGGAACGTCACGTGC-3' and reverse, 5'-AAG AGGAGGCCAGCCAGACA-3' for osterix; forward, 5'-CTC AGGGTTTCAGTGGTT-3' and reverse, 5'-TTTCCACGA GCACCCATC-3' for collagen type $1, \alpha 1$ chain (Col1 $\alpha 1)$; and forward, 5'-ACAGGGGAGGTGATAGCATT-3' and reverse, 5'-GACCAAAAGCCTTCATACATCTC-3' for GAPDH.

Western blot analysis. The tibias were homogenized and extracted in NP-40 buffer, followed by 5-10 min boiling and centrifugation $\left(12,000 \mathrm{x}\right.$ g for $15 \mathrm{~min}$ at $\left.4^{\circ} \mathrm{C}\right)$ to obtain the supernatant. Samples containing $50 \mu \mathrm{g}$ protein were separated by $10 \%$ SDS-PAGE and transferred onto nitrocellulose membranes (Bio-Rad Laboratories, Inc., Hercules, CA, USA). The membranes were incubated at room temperature for $4 \mathrm{~h}$ with primary cathepsin K antibodies (1:500; R\&D Systems). The blots were washed 3 times with TBS with Tween-20 (TBST), and incubated with donkey anti-goat immunoglobulin G (IgG; cat. no. OARA01784) and donkey anti-mouse IgG secondary antibodies (cat. no. OARA01848) at room temperature for $2 \mathrm{~h}$, conjugated to IRDye $800 \mathrm{CW}$ Infrared Dye (LI-COR Biosciences, Lincoln, NE, USA), diluted 1:10,000. The membranes were washed 3 times with TBST. Blots were visualized using the Odyssey Infrared Imaging System (LI-COR Biosciences) and normalized to $\beta$-actin expression, to correct for unequal loading, using a mouse monoclonal anti- $\beta$-actin antibody (cat. no. AP0060; Bioworld Technology, Inc., St. Louis Park, MN, USA).

Statistical analysis. Data are presented as the mean \pm standard deviation for each group. All statistical analyses were performed using PRISM version 4.0 (GraphPad Software, Inc., La Jolla, CA, USA). Inter-group differences were analyzed by one-way analysis of variance, followed by a post hoc Tukey's honest significant difference test for multiple comparisons. $\mathrm{P}<0.05$ was considered to indicate a statistically significant difference. 
A
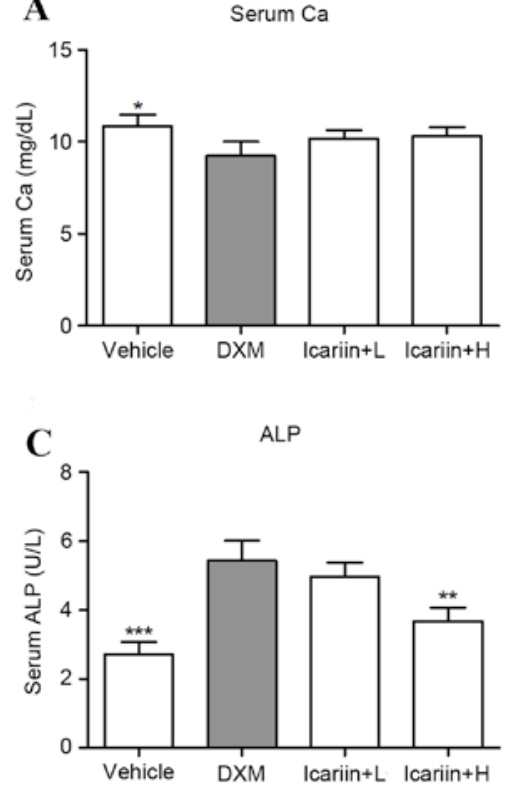

E

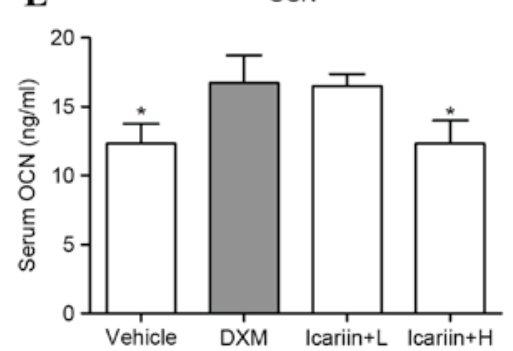

B

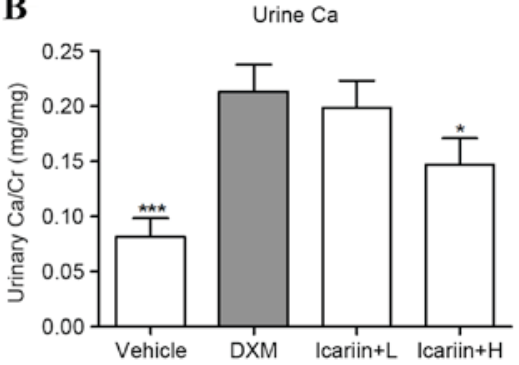

D

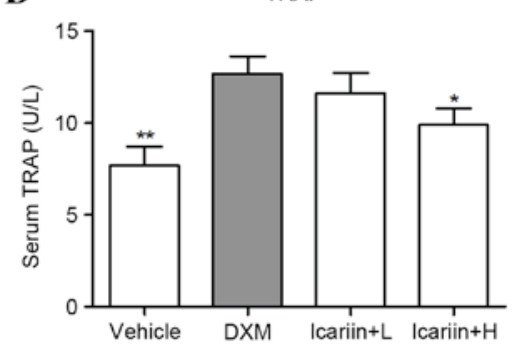

F

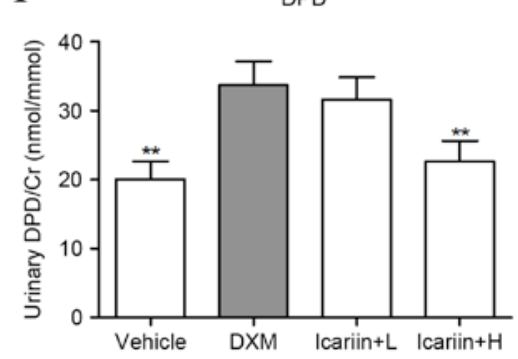

Figure 1. Effects of icariin on physiological and biochemical parameters. (A) Serum and (B) urine Ca were measured by standard colorimetric methods. Serum levels of (C) ALP, (D) TRAP, (E) OCN and (F) urine levels of DPD were detected by ELISA. Data are expressed as mean \pm standard deviation (n=12/group). ${ }^{*} \mathrm{P}<0.05,{ }^{* *} \mathrm{P}<0.01$ and ${ }^{* * *} \mathrm{P}<0.001$ vs. DXM group. Ca, calcium; Cr, creatinine; ALP, alkaline phosphatase; TRAP, tartrate resistant acid phosphatase; OCN, osteocalcin; DPD, deoxypyridinoline; DXM, dexamethasone; L, low dose; H, high dose; U, units.

\section{Results}

Effects of icariin on physiological and biochemical properties. Administration of DXM significantly downregulated the serum Ca level, compared with the control group, and icariin treatment did not recover these levels in GIOP mice (Fig. 1A). In contrast, DXM exposure markedly increased the level of urine $\mathrm{Ca}$; however, high dose icariin-treatment reversed this upregulation in GIOP mice (Fig. 1B). ELISA analysis of bone formation and resorption markers indicated that DXM induced high bone turnover in mice, as evidenced by a significant increase in serum ALP (Fig. 1C), TRAP (Fig. 1D) OCN (Fig. 1E) and urinary DPD/Cr (Fig. 1F) levels. However, mice in the high dose icariin $+\mathrm{H}$ group demonstrated decreased serum ALP, TRAP and OCN and urinary DPD/Cr.

Effects of icariin on bone microarchitecture and biomechanical parameters. BMD decreased by 27.73 and $42.59 \%$ at weeks 6 and 12, respectively, following administration of DXM $(\mathrm{P}<0.001)$, whereas administration with high-dose icariin $(100 \mathrm{mg} / \mathrm{kg})$ significantly restored the DXM-induced reduction in BMD in GIOP mice (Fig. 2A). A gross decrease in bone mass was observed following 12 weeks of DXM administration, which was inhibited when icariin was co-administered
(Fig. 2B). Furthermore, structural parameters of the trabecular bone network in the tibial proximal metaphysis were measured. The results demonstrated that DXM-treated mice demonstrated significant decreases in trabecular BV/TV, Tb.N and Tb.Th, and increases in Tb.Sp, compared with the control group (Fig. 2C). Notably, icariin treatment in GIOP mice reversed the DXM-induced bone microarchitectural deteriorations. Bone biomechanical parameters, including maximum load and stiffness, decreased by 61.24 and $45.83 \%$ respectively, following DXM treatment. Mice in the icarrin $+\mathrm{H}$ group demonstrated significantly restoration of the DXM-induced biomechanical alterations in the tibial proximal metaphysis (Fig. 2D).

Effects of icariin on the mRNA expression of key regulators in bone metabolism. ALP and TRAP mRNA were significantly increased in the DXM group, compared with the control group (Fig. 3A and B); however, these were reduced by high-dose icariin co-administration. Furthermore, expression of the bone formation-associated genes Runx2, osterix and Colla1 were decreased with DXM administration compared with the vehicle group, and this was prevented by high dose icariin treatment in DXM-induced GIOP mice (Fig. 3C). To further investigate the molecular mechanism underlying DXM-induced inhibition of bone formation and promotion 

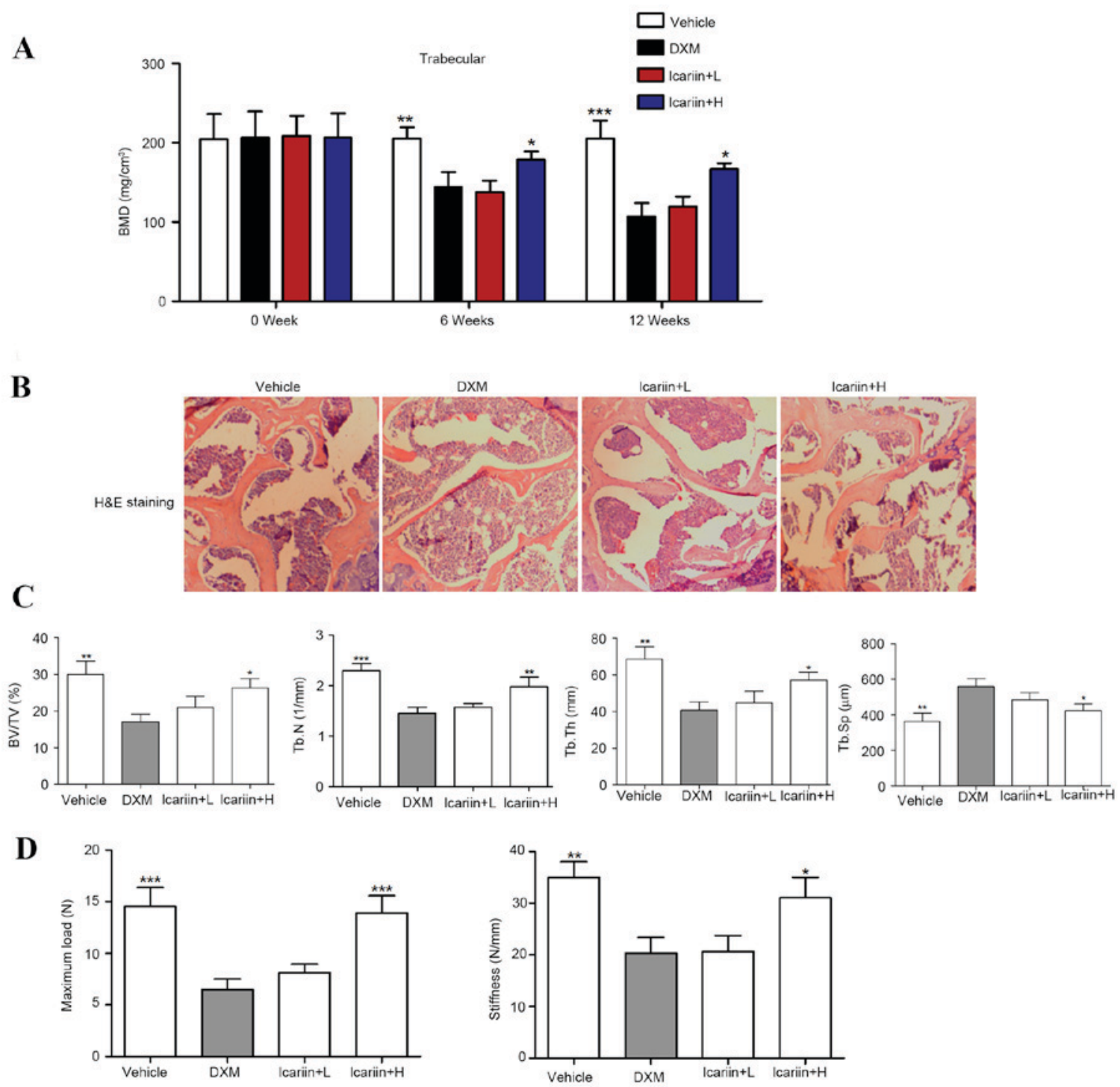

Figure 2. Effects of icariin on bone microarchitecture and biomechanical parameters. (A) Volumetric BMD was measured by micro-CT. (B) Hematoxylin and eosin staining of the trabecular bone zone below the growth plate of the tibial proximal metaphysis (magnification, x50). (C) BV/TV, Tb.N, Tb.Th and Tb.Sp were assessed by micro-CT. (D) Maximum load and stiffness were assessed by micro-force testing. Data are expressed as mean \pm standard deviation ( $\mathrm{n}=12$ /group). ${ }^{*} \mathrm{P}<0.05,{ }^{* *} \mathrm{P}<0.01$ and ${ }^{* * * *} \mathrm{P}<0.001$ vs. DXM group. BMD, bone mineral density; BV/TV, bone volume over total volume; Tb.N, trabecula number; Tb.Th, trabecula thickness; Tb.Sp, trabecula separation; DXM, dexamethasone; L, low dose; H, high dose; N, Newtons; CT, computed tomography.

of bone resorption, RT-qPCR was used to assess the osteoclastogenesis-associated genes RANKL and OPG, which are produced by the osteoblast. The results indicated that DXM significantly elevated the expression of RANKL, and significantly reduced the RANKL/OPG ratio. However, these alterations in RANKL expression and the RANKL/OPG ratio were reversed by icariin co-administration (Fig. 3D).

Effects of icariin on cathepsin $K$ and miR-186 expression. Cathepsin K mRNA and protein expression levels were significantly upregulated in DXM-treated mice, compared with the control group (Fig. 4A and B). However, these were reversed when icariin was co-administered in GIOP mice. Furthermore, the tibial levels of miR-186 were suppressed in GIOP mice compared with the control group; however, these levels were increased in icariin-supplemented GIOP mice (Fig. 4C). Notably, Spearman's rank correlation analysis indicated that the expression levels of cathepsin $\mathrm{K}$ protein and
miR-186 were inversely correlated in DXM and icariin $+\mathrm{H}$ group mice (Fig. 4D). To further investigate the effect of cathepsin $\mathrm{K}$ on cartilaginous degeneration, safranin $\mathrm{O}$ staining was performed to observe the upper epiphyseal cartilage in the proximal tibias. The thickness of cartilage adjacent to the epiphyseal plate was reduced in the proximal tibia of the DXM mice, suggesting that DXM induces a delayed formation of new cartilage on the upper epiphyseal plate. However, the thickness of newly formed cartilage of the GIOP mice was improved by high concentrations of icariin (Fig. 4E).

Cathepsin $K$ is a direct target of miR-186 in osteoclasts. The target prediction tools TargetScan, PicTar and microRNA. org were used to identify potential target genes of miR-186; the results indicated that miR-186 may regulate cathepsin $\mathrm{K}$ (Fig. 5A). To investigate whether miR-186 may directly regulate cathepsin $\mathrm{K}$ expression, a luciferase reporter construct containing the 3'-UTR of cathepsin K was used. The wild-type 

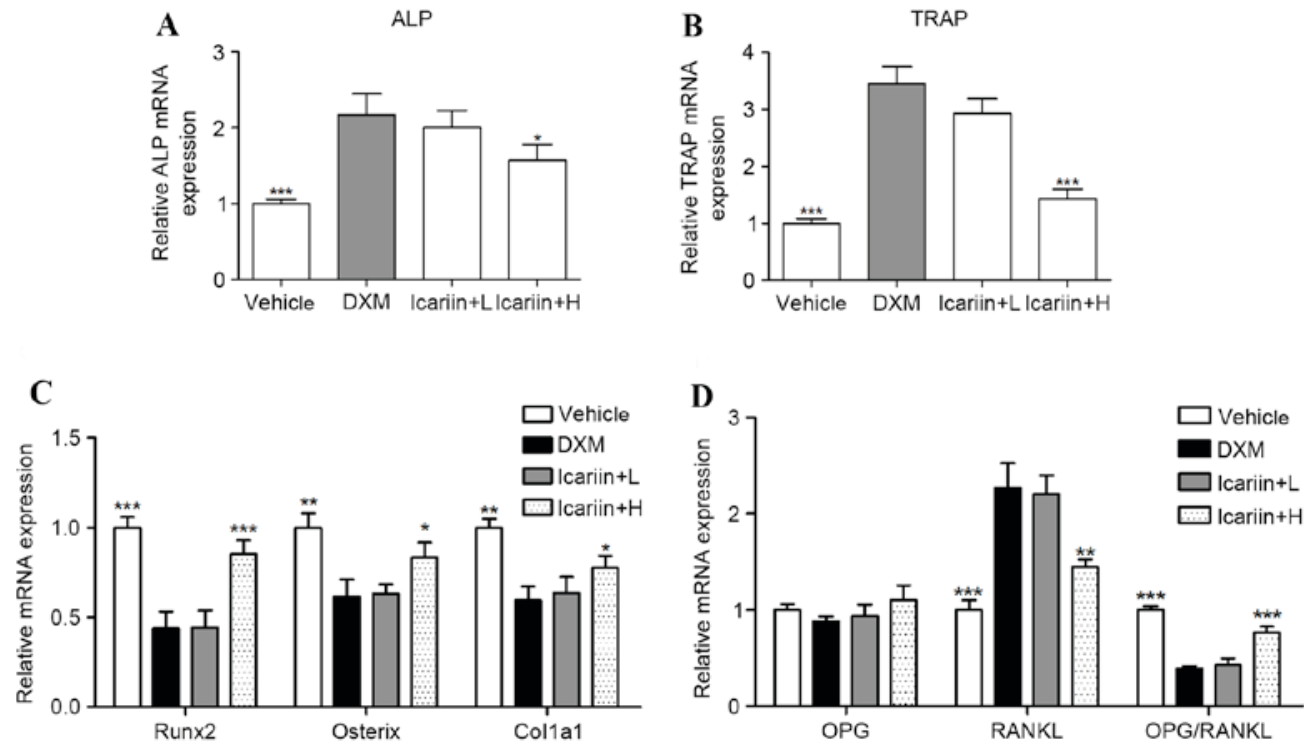

Figure 3. Effects of icariin on the mRNA expression of key regulators in bone metabolism. The mRNA expression of (A) ALP and (B) TRAP were measured by reverse transcription-quantitative polymerase chain reaction. The mRNA expression of (C) Runx2, Osterix and Colla1, and (D) OPG and RANKL were measured by RT-qPCR. Data are expressed as mean \pm standard deviation ( $\mathrm{n}=$ /group). ${ }^{*} \mathrm{P}<0.05,{ }^{* * *} \mathrm{P}<0.01$ and ${ }^{* * * *} \mathrm{P}<0.001$ vs. DXM group. ALP, alkaline phosphatase; TRAP, tartrate resistant acid phosphatase; Runx2, runt related transcription factor 2; Colla1, collage type $1 \propto 1$ chain; OPG, osteoprotegerin; RANKL, receptor activator of nuclear factor $\kappa \mathrm{B}$ ligand; $\mathrm{DXM}$, dexamethasone; $\mathrm{L}$, low dose; $\mathrm{H}$, high dose.

A

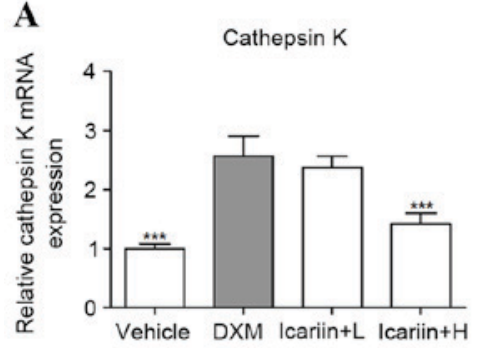

C

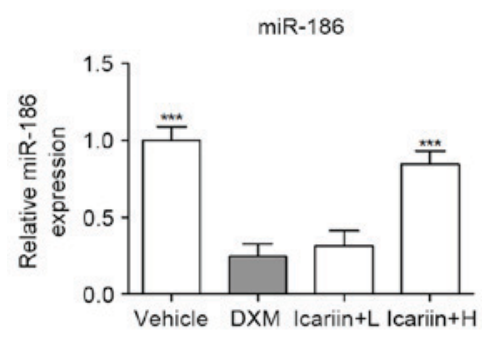

B

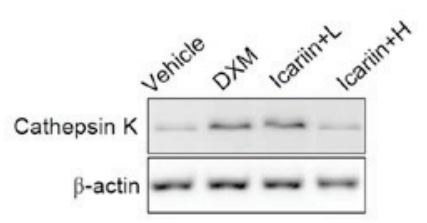

\section{D}

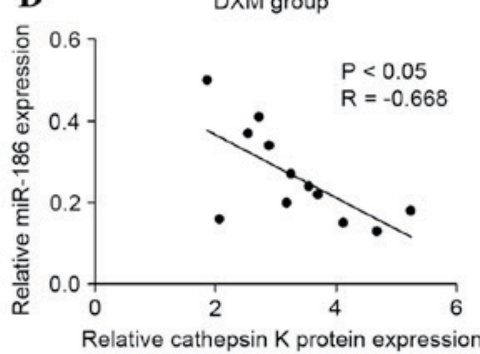

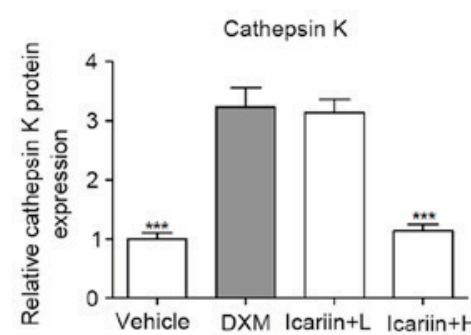

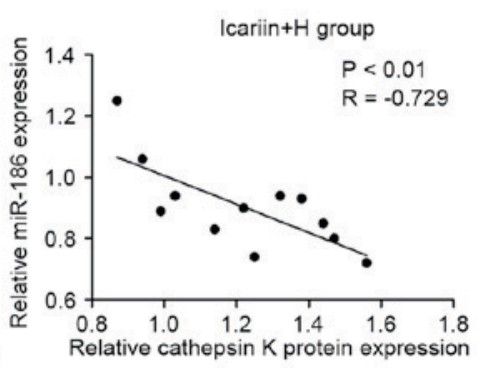

E

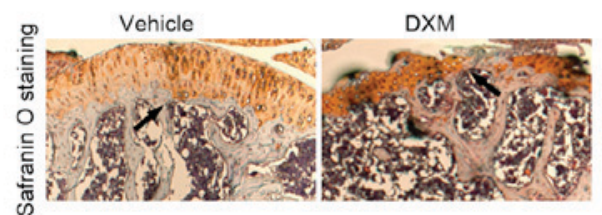

Icariin+L

Icariin+H

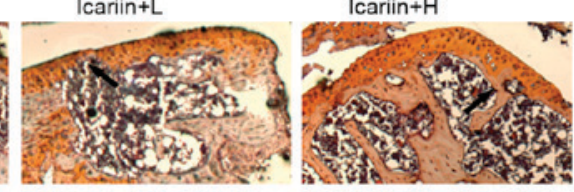

Figure 4. Effects of icariin on cathepsin K and miR-186 expression. The (A) mRNA and (B) protein expression levels of cathepsin K and (C) miR-186 were measured in the tibias. (D) Spearman's rank correlation analysis between the expression levels of cathepsin K protein and miR-186 were inversely correlated in DXM and icariin + H group mice. (E) Adjacent cartilage was visualized by Safranin O staining of the proximal metaphysis of the tibia (magnification, x50). Data are expressed as mean \pm standard deviation $\left(\mathrm{n}=12\right.$ /group). ${ }^{* * * *} \mathrm{P}<0.001$ vs. DXM group. DXM, dexamethasone; $\mathrm{L}$, low dose; H, high dose; miR, microRNA.

and mutant cathepsin $\mathrm{K}$ luciferase expression vectors were transfected alongside an miR-186 mimic into HEK293T, and the level of luciferase enzyme activity was measured. Overexpression of miR-186 suppressed the luciferase activity of the reporter gene; however, a mutation in the miRNA binding site abolished the luciferase activity repression, confirming that the cathepsin K 3'-UTR contains a seed site for miR-186 (Fig. 5B).

Transfection with an miR-186 mimic decreased the levels of cathepsin $\mathrm{K}$ in osteoclasts, whereas transfection with 
A

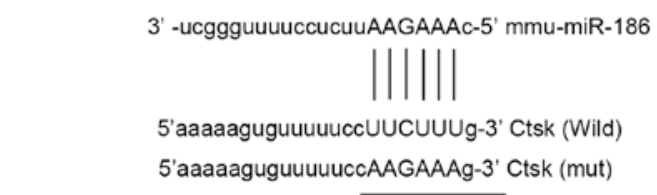

C
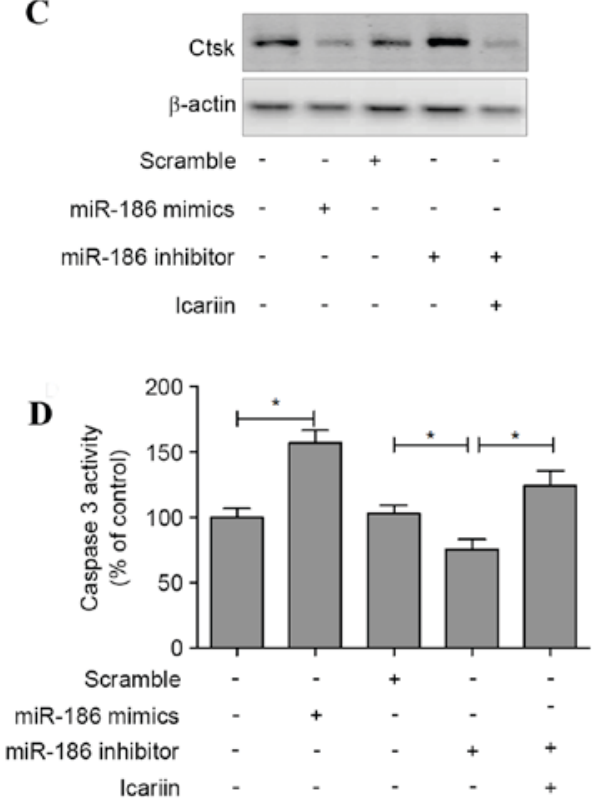

B
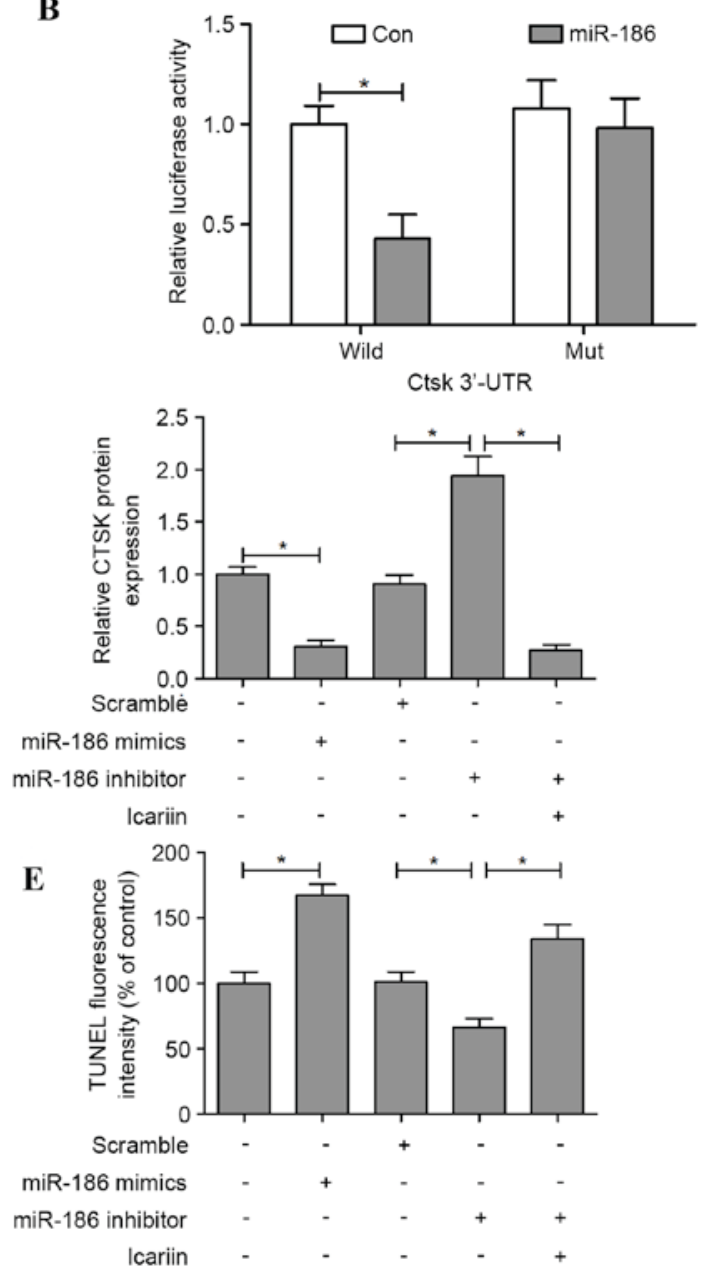

Figure 5. Cathepsin K is a direct target of miR-186 in osteoclasts. (A) Schematic representation of the putative miR-186 binding site in the cathepsin K 3'-UTR and (B) luciferase activity assay. (C) The protein expression of cathepsin K was measured by western blotting in osteoclasts transfected with an miR-186 mimic or inhibitor for $48 \mathrm{~h}$. (D) Caspase-3 activity assay and (E) TUNEL staining were performed following osteoclast transfection with miR-186 mimics or inhibitor for 48 h. Data are expressed as mean \pm standard deviation ( $\mathrm{n}=12 /$ group). * $\mathrm{P}<0.05$ vs. DXM group. 3'-UTR, 3'-untranslated region; miR, microRNA; TUNEL, triphosphate nick-end labeling; Ctsk, cathepsin K; mut, mutant.

miR-186 inhibitor increased these levels. However, cathepsin K protein expression was reduced when icariin was administered alongside the miR-186 inhibitor (Fig. 5C). In addition, apoptotic features induced by transfection with miR-186 mimic or inhibitor were assessed. The results demonstrated that caspase-3 activity was significantly increased in the miR-186 mimic group compared with the control group. In contrast, osteoclasts transfected with an miR-186 inhibitor suppressed caspase- 3 activity, and this downregulation of caspase- 3 was reversed when icariin was co-administered with the miR-186 inhibitor (Fig. 5D). TUNEL staining indicated that transfection with the miR-186 mimic induced cell apoptosis, whereas the miR-186 inhibitor suppressed cell apoptosis, and icariin treatment reversed the suppression of cell apoptosis in miR-186 inhibitor-treated osteoclasts (Fig. 5E).

\section{Discussion}

The present study revealed that cathepsin $\mathrm{K}$ is upregulated in GIOP mice, and this is characterized by a reduction in bone volume and strength, accompanied with a high bone turnover, as evidenced by a significant increase in bone formation and resorption markers. Furthermore, when the expression of cathepsin $\mathrm{K}$ was blocked by icariin, the bone abnormalities were normalized. Notably, the protective effects of icariin against bone deteriorations in GIOP mice involved activation of miR-186, which serves to suppress cathepsin $\mathrm{K}$.

It has previously been suggested that glucocorticoid treatment may influence bone remodeling and calcium homeostasis (27). Recent studies have indicated that DXM-exposure exhibits the typical features of GIOP in rabbits or mice, evidenced by the alterations in the basic bone microarchitecture and biomechanical parameters $(2,28)$. A study investigating the pharmacological mechanisms of icariin revealed multiple targets in bone metabolism, and cathepsin $\mathrm{K}$ was identified as the critical node in the associated signaling networks (13). Growing evidence has suggested that cathepsin $\mathrm{K}$ inhibition may specifically block bone resorption without interfering with other pathways $(16,29,30)$. In a randomized trial, the cathepsin $\mathrm{K}$ inhibitor odanacatib demonstrated an ability to decrease bone resorption, maintain bone formation and increase areal and volumetric BMD and increase estimated bone strength, in the hip and spine (31). 
Icariin has been identified as an inhibitor of cathepsin $\mathrm{K}$ and may suppress cartilage and bone degradation in mice with collagen-induced arthritis (13). In vivo, icariin has demonstrated significant anti-osteoporosis effects in ovariectomized rats and mice, glucocorticoid-induced rats and OPG knockout mice $(7,8)$. However, in vitro studies have indicated that icariin may reduce TRAP activity and increase osteogenic differentiation, calcium deposition and mineralized nodule formation in induced bone marrow stromal and RAW264.7 cells $(32,33)$. The present study revealed that high concentrations of icariin inhibits DXM-induced high bone turnover and cathepsin $\mathrm{K}$ upregulation in GIOP mice.

Several miRNAs have been associated with glucocorticoid-induced osteogenic differentiation and bone deterioration (34). Specifically, miR-29a ameliorates glucocorticoid-induced suppression of osteoblast differentiation by regulating $\beta$-catenin acetylation (35), and miR-29a overexpression may represent an alternative strategy for alleviating glucocorticoid-induced bone deterioration (36). Furthermore, miR-216a reverses DXM suppression of osteogenesis, promotes osteoblast differentiation and enhances bone formation by regulating the c-Casitas B-lineage lymphoma-mediated phosphoinositide 3-kinase/protein kinase B pathway (37). The results of the present study suggested that icariin prevents DXM-induced bone loss by inhibiting activated cathepsin $\mathrm{K}$ and increasing miR-186 levels. Notably, the expression levels of cathepsin K protein and miR-186 were inversely correlated in DXM and icariin + $\mathrm{H}$ group mice, and bioinformatics analysis suggested that miR-186 may regulate cathepsin $\mathrm{K}$ via binding to a seed region in the 3'-UTR of cathepsin K. These results suggested that miR-186 and cathepsin K serve a role in icariin-mediated protection against DXM-induced bone deterioration.

In conclusion, the present study demonstrated that icariin may significantly ameliorate bone deterioration in GIOP mice, and the underlying mechanism may be mediated, at least partially, via activation of miR-186 and subsequent suppression of cathepsin $\mathrm{K}$. These results may facilitate understanding of the underlying molecular mechanisms in DXM-induced osteoporosis, and may provide evidence to support the use of icariin as a therapeutic approach in the management of glucocorticoid-induced bone loss, and the disequilibrium of calcium homeostasis.

\section{References}

1. Spreafico A, Frediani B, Francucci CM, Capperucci C, Chellini F and Galeazzi M: Role of apoptosis in osteoporosis induced by glucocorticoids. J Endocrinol Invest 31 (Suppl 7): S22-S27, 2008.

2. Yongtao Z, Kunzheng W, Jingjing Z, Hu S, Jianqiang K, Ruiyu L and Chunsheng W: Glucocorticoids activate the local renin-angiotensin system in bone: Possible mechanism for glucocorticoid-induced osteoporosis. Endocrine 47: 598-608, 2014.

3. Hofbauer LC, Gori F, Riggs BL, Lacey DL, Dunstan CR, Spelsberg TC and Khosla S: Stimulation of osteoprotegerin ligand and inhibition of osteoprotegerin production by glucocorticoids in human osteoblastic lineage cells: Potential paracrine mechanisms of glucocorticoid-induced osteoporosis. Endocrinology 140: 4382-4389, 1999.

4. Shaker JL and Lukert BP: Osteoporosis associated with excess glucocorticoids. Endocrinol Metab Clin North Am 34: 341-356, 2005.

5. Li C, Li Q, Mei Q and Lu T: Pharmacological effects and pharmacokinetic properties of icariin, the major bioactive component in Herba Epimedii. Life Sci 126: 57-68, 2015.
6. Zhang X, Liu T, Huang Y, Wismeijer D and Liu Y: Icariin: Does it have an osteoinductive potential for bone tissue engineering? Phytother Res 28: 498-509, 2014.

7. Yang L, Yu Z, Qu H and Li M: Comparative effects of hispidulin, genistein, and icariin with estrogen on bone tissue in ovariectomized rats. Cell Biochem Biophys 70: 485-490, 2014.

8. Liu M, Zhong C, He RX and Chen LF: Icariin associated with exercise therapy is an effective treatment for postmenopausal osteoporosis. Chin Med J (Engl) 125: 1784-1789, 2012.

9. Feng R, Feng L, Yuan Z, Wang D, Wang F, Tan B, Han S, Li T, Li D and Han Y: Icariin protects against glucocorticoid-induced osteoporosis in vitro and prevents glucocorticoid-induced osteocyte apoptosis in vivo. Cell Biochem Biophys 67: 189-197, 2013.

10. Zhang G, Qin L and Shi Y: Epimedium-derived phytoestrogen flavonoids exert beneficial effect on preventing bone loss in late postmenopausal women: A 24-month randomized, double-blind and placebo-controlled trial. J Bone Miner Res 22: 1072-1079, 2007.

11. Song L, Zhao J, Zhang X, Li H and Zhou Y: Icariin induces osteoblast proliferation, differentiation and mineralization through estrogen receptor-mediated ERK and JNK signal activation. Eur J Pharmacol 714: 15-22, 2013.

12. Wu Y, Xia L, Zhou Y, Xu Y and Jiang X: Icariin induces osteogenic differentiation of bone mesenchymal stem cells in a MAPK-dependent manner. Cell Prolif 48: 375-384, 2015.

13. Sun P, Liu Y, Deng X, Yu C, Dai N, Yuan X, Chen L, Yu S, Si W, Wang X, et al: An inhibitor of cathepsin K, icariin suppresses cartilage and bone degradation in mice of collagen-induced arthritis. Phytomedicine 20: 975-979, 2013.

14. Skoumal M, Haberhauer G, Kolarz G, Hawa G, Woloszczuk W, Klingler A, Varga F and Klaushofer K: The imbalance between osteoprotegerin and cathepsin $\mathrm{K}$ in the serum of patients with longstanding rheumatoid arthritis. Rheumatol Int 28: 637-641, 2008.

15. Lewiecki EM: Odanacatib, a cathepsin K inhibitor for the treatment of osteoporosis and other skeletal disorders associated with excessive bone remodeling. IDrugs 12: 799-809, 2009.

16. Bone HG, McClung MR, Roux C, Recker RR, Eisman JA, Verbruggen N, Hustad CM, DaSilva C, Santora AC and Ince BA: Odanacatib, a cathepsin-K inhibitor for osteoporosis: A two-year study in postmenopausal women with low bone density. J Bone Miner Res 25: 937-947, 2010.

17. Dole NS and Delany AM: MicroRNA variants as genetic determinants of bone mass. Bone 84: 57-68, 2016.

18. Kagiya T: MicroRNAs and osteolytic bone metastasis: The roles of microRNAs in tumor-induced osteoclast differentiation. J Clin Med 4: 1741-1752, 2015.

19. Xia Z, Chen C, Chen P, Xie H and Luo X: MicroRNAs and their roles in osteoclast differentiation. Front Med 5: 414-419, 2011.

20. Arfat Y, Xiao WZ, Ahmad M, Zhao F, Li DJ, Sun YL, Hu L, Zhihao C, Zhang G, Iftikhar S, et al: Role of microRNAs in osteoblasts differentiation and bone disorders. Curr Med Chem 22: 748-758, 2015.

21. Gamez B, Rodriguez-Carballo E and Ventura F: MicroRNAs and post-transcriptional regulation of skeletal development. J Mol Endocrinol 52: R179-R197, 2014.

22. Taipaleenmäki H, Bjerre Hokland L, Chen L, Kauppinen S and Kassem M: Mechanisms in endocrinology: micro-RNAs: Targets for enhancing osteoblast differentiation and bone formation. Eur J Endocrinol 166: 359-371, 2012.

23. Cuetara BL, Crotti TN, O'Donoghue AJ and McHugh KP: Cloning and characterization of osteoclast precursors from the RAW264.7 cell line. In Vitro Cell Dev Biol Anim 42: 182-188, 2006.

24. Parfitt AM, Drezner MK, Glorieux FH, Kanis JA, Malluche H, Meunier PJ, Ott SM and Recker RR: Bone histomorphometry: Standardization of nomenclature, symbols and units. Report of the ASBMR Histomorphometry Nomenclature Committee. J Bone Miner Res 2: 595-610, 1987.

25. Turner $\mathrm{CH}$ and Burr DB: Basic biomechanical measurements of bone: A tutorial. Bone 14: 595-608, 1993.

26. Livak KJ and Schmittgen TD: Analysis of relative gene expression data using real-time quantitative PCR and the 2(-Delta Delta C(T)) method. Methods 25: 402-408, 2001.

27. Jensen PR, Andersen TL, Hauge EM, Bollerslev J and Delaisse JM: A joined role of canopy and reversal cells in bone remodeling-lessons from glucocorticoid-induced osteoporosis. Bone 73: 16-23, 2014.

28. Tamura Y, Kawao N, Yano M, Okada K, Okumoto K, Chiba Y, Matsuo $\mathrm{O}$ and Kaji H: Role of plasminogen activator inhibitor-1 in glucocorticoid-induced diabetes and osteopenia in mice. Diabetes 64: 2194-2206, 2015. 
29. Panwar $P$, Soe $K$, Guido RV, Bueno RV, Delaisse JM and Bromme D: A novel approach to inhibit bone resorption: Exosite inhibitors against cathepsin K. Br J Pharmacol 173: 396-410, 2016.

30. Helali AM, Iti FM and Mohamed IN: Cathepsin K inhibitors: A novel target but promising approach in the treatment of osteoporosis. Curr Drug Targets 14: 1591-1600, 2013.

31. Brixen K, Chapurlat R, Cheung AM, Keaveny TM, Fuerst T, Engelke K, Recker R, Dardzinski B, Verbruggen N, Ather S, et al: Bone density, turnover, and estimated strength in postmenopausal women treated with odanacatib: A randomized trial. J Clin Endocrinol Metab 98: 571-580, 2013.

32. Fan JJ, Cao LG, Wu T, Wang DX, Jin D, Jiang S, Zhang ZY, Bi L and Pei GX: The dose-effect of icariin on the proliferation and osteogenic differentiation of human bone mesenchymal stem cells. Molecules 16: 10123-10133, 2011.

33. Cui J, Zhu M, Zhu S, Wang G, Xu Y and Geng D: Inhibitory effect of icariin on Ti-induced inflammatory osteoclastogenesis. J Surg Res 192: 447-453, 2014.

34. Li T, Li H, Li T, Fan J, Zhao RC and Weng X: MicroRNA expression profile of dexamethasone-induced human bone marrow-derived mesenchymal stem cells during osteogenic differentiation. J Cell Biochem 115: 1683-1691, 2014.
35. Ko JY, Chuang PC, Chen MW, Ke HC, Wu SL, Chang YH, Chen YS and Wang FS: MicroRNA-29a ameliorates glucocorticoid-induced suppression of osteoblast differentiation by regulating $\beta$-catenin acetylation. Bone 57: 468-475, 2013.

36. Wang FS, Chuang PC, Lin CL, Chen MW, Ke HJ, Chang YH, Chen YS, Wu SL and Ko JY: MicroRNA-29a protects against glucocorticoid-induced bone loss and fragility in rats by orchestrating bone acquisition and resorption. Arthritis Rheum 65: 1530-1540, 2013.

37. Li H, Li T, Fan J, Li T, Fan L, Wang S, Weng X, Han Q and Zhao RC: miR-216a rescues dexamethasone suppression of osteogenesis, promotes osteoblast differentiation and enhances bone formation, by regulating c-Cbl-mediated PI3K/AKT pathway. Cell Death Differ 22: 1935-1945, 2015.

This work is licensed under a Creative Commons Attribution-NonCommercial-NoDerivatives 4.0 International (CC BY-NC-ND 4.0) License. 